Pacific

Journal of

Mathematics

SCHWARZIAN DERIVATIVES

AND A LINEARLY INVARIANT FAMILY IN $\mathbb{C}^{n}$

RODRIGO HERNÁNDEZ R.

Volume $228 \quad$ No. 2

December 2006 


\title{
SCHWARZIAN DERIVATIVES AND A LINEARLY INVARIANT FAMILY IN $\mathbb{C}^{n}$
}

\author{
RODRIGO HERNÁNDEZ R.
}

\begin{abstract}
We use Oda's definition of the Schwarzian derivative for locally univalent holomorphic maps $F$ in several complex variables to define a Schwarzian derivative operator $\mathscr{S} F$. We use the Bergman metric to define a norm $\|\mathscr{S} \boldsymbol{F}\|$ for this operator, which in the ball is invariant under composition with automorphisms. We study the linearly invariant family
\end{abstract}

$$
\mathscr{F}_{\alpha}=\left\{F: \mathbb{B}^{n} \rightarrow \mathbb{C}^{n} \mid F(0)=0, D F(0)=I d,\|\mathscr{F}\| \leq \alpha\right\},
$$

estimating its order and norm order.

\section{Introduction}

The link between the Schwarzian derivative of a locally univalent holomorphic map in one complex variable, given by

$$
S f=\left(\frac{f^{\prime \prime}}{f^{\prime}}\right)^{\prime}-\frac{1}{2}\left(\frac{f^{\prime \prime}}{f^{\prime}}\right)^{2}
$$

with the univalence of $f$ and distortion problems has been studied extensively; see [Chuaqui and Osgood 1993; Epstein 1986; Kraus 1932; Nehari 1949], for example. $S f$ vanishes identically if and only if $f$ is a Möbius mapping, and we have $S(f \circ g)=(S f \circ g)\left(g^{\prime}\right)^{2}+S g$. An analytic function $f$ with Schwarzian derivative $S f=2 p$ has the form $f=u / v$, where $u$ and $v$ are any linearly independent solutions of the equation $u^{\prime \prime}+p u=0$. If $f$ is defined in the unit disk $\mathbb{D}$, the norm

$$
\|S f\|=\sup _{|z|=1}\left(1-|z|^{2}\right)^{2}|S f(z)|
$$

is invariant under precomposition with automorphisms of the disk.

Some analogues of the Schwarzian derivative in several complex variables are available, but results relating it to the aforementioned problems of univalence and

MSC2000: primary 32A17, 32W50; secondary 32H02, $30 \mathrm{C} 35$.

Keywords: Several complex varaibles, Schwarzian derivative, Linearly invariant families, Sturm comparison. 
distortion are less satisfactory than in one variable [Molzon and Pinney Mortensen 1997]. Consider the overdetermined system of partial differential equations

$$
\frac{\partial^{2} u}{\partial z_{i} \partial z_{j}}=\sum_{k=1}^{n} P_{i j}^{k}(z) \frac{\partial u}{\partial z_{k}}+P_{i j}^{0}(z) u, \quad i, j=1,2, \ldots, n,
$$

where $z=\left(z_{1}, z_{2}, \ldots, z_{n}\right) \in \mathbb{C}^{n}$. The system is called completely integrable if (1-1) has $n+1$ linearly independent solutions. The system (1-1) is said to be in canonical form (see [Yoshida 1976]) if the coefficients satisfy

$$
\sum_{j=1}^{n} P_{i j}^{j}(z)=0, \quad i=1,2, \ldots, n .
$$

T. Oda [1974] defined the Schwarzian derivative $S_{i j}^{k}$ of a locally injective holomorphic mapping $F\left(z_{1}, z_{2}, \ldots, z_{n}\right)=\left(w_{1}, w_{2}, \ldots, w_{n}\right)$ as

$$
S_{i j}^{k} F=\sum_{l=1}^{n} \frac{\partial^{2} w_{l}}{\partial z_{i} \partial z_{j}} \frac{\partial z_{k}}{\partial w_{l}}-\frac{1}{n+1}\left(\delta_{i}^{k} \frac{\partial}{\partial z_{j}}+\delta_{j}^{k} \frac{\partial}{\partial z_{i}}\right) \log \Delta,
$$

where $i, j, k=1,2, \ldots, n, \Delta=\operatorname{det}(\partial F / \partial z)$, and $\delta_{i}^{k}$ is the Kronecker symbol. For $n>1$ these Schwarzian derivatives satisfy

$$
S_{i j}^{k} F=0 \text { for all } i, j, k=1,2, \ldots, n
$$

if and only if $F(z)$ is a Möbius transformation, that is, if it has the form

$$
F(z)=\left(\frac{l_{1}(z)}{l_{0}(z)}, \ldots, \frac{l_{n}(z)}{l_{0}(z)}\right),
$$

where $l_{i}(z)=a_{i 0}+a_{i 1} z_{1}+\cdots+a_{i n} z_{n}$ with $\operatorname{det}\left(a_{i j}\right) \neq 0$. For a composition we have

$$
S_{i j}^{k}(G \circ F)(z)=S_{i j}^{k} F(z)+\sum_{l, m, r=1}^{n} S_{l m}^{r} G(w) \frac{\partial w_{l}}{\partial z_{i}} \frac{\partial w_{m}}{\partial z_{j}} \frac{\partial z_{k}}{\partial w_{r}}, w=F(z) .
$$

Thus, precomposition with a Möbius transformation $G$ leads to $S_{i j}^{k}(G \circ F)=S_{i j}^{k} F$. The coefficients $S_{i j}^{0} F$ are given by

$$
S_{i j}^{0} F(z)=\Delta^{1 /(n+1)}\left(\frac{\partial^{2}}{\partial z_{i} \partial z_{j}} \Delta^{-1 /(n+1)}-\sum_{k=1}^{n} \frac{\partial}{\partial z_{k}} \Delta^{-1 /(n+1)} S_{i j}^{k} F(z)\right) .
$$

The function $u=\Delta^{-1 / n+1}$ is always a solution of (1-1) with $S_{i j}^{k} F=P_{i j}^{k}$.

Remark 1.1. For $n=1, S_{11}^{1} f=0$ for all locally injective $f$, but $S_{11}^{0} f=-\frac{1}{2} S f$. 
Proposition 1.2 [Yoshida 1976]. Let (1-1) be a completely integrable system in canonical form and consider a set $u_{0}(z), u_{1}(z), \ldots, u_{n}(z)$ of linearly independent solutions. Then

$$
P_{i j}^{k}(z)=S_{i j}^{k} F(z), \quad i, j, k=1,2, \ldots, n,
$$

where $F(z)=\left(w_{1}(z), \ldots, w_{n}(z)\right)$ and $w_{i}(z)=u_{i}(z) / u_{0}(z)$.

Remark 1.3. In contrast to the one-dimensional case, when $n>1$ the Schwarzian derivatives $S_{i j}^{k} F$ are differential operators of order 2 . One way to understand this phenomenon is through a dimensional argument: For $n=1$ the Möbius group has dimension 3 , which allows one to choose $f\left(z_{0}\right), f^{\prime}\left(z_{0}\right)$ and $f^{\prime \prime}\left(z_{0}\right)$ for a holomorphic mapping $f$ at a given point $z_{0}$ arbitrarily. It would therefore be pointless to seek a Möbius-invariant differential operator of order 2. But for $n>1$ the number of parameters involved in the value and all derivatives of order 1 and 2 of a locally biholomorphic mapping is $n^{2}(n+1) / 2+n^{2}+n$, which exceeds the dimension $n^{2}+2 n$ of the corresponding Möbius group in $\mathbb{C}^{n}$. Moreover, since $S_{i j}^{k} F=S_{j i}^{k} F$ for all $k$ and

$$
\sum_{j=1}^{n} S_{i j}^{j} F=0
$$

there are exactly $n(n-1)(n+2) / 2$ independent terms $S_{i j}^{k} F$, which is equal to the excess mentioned above.

In this paper we employ the Oda Schwarzian derivatives $S_{i j}^{k}$ to propose a $S c h w a r$ zian derivative operator $\mathscr{Y} F$. Using the Bergman metric, we will define a norm for $\mathscr{S} F$, which for mappings defined in the ball $\mathbb{B}$ turns out to be invariant under the group of automorphisms. We then focus on the study of geometric properties of the linearly invariant family given by bounded Schwarzian norm. We will appeal to the relationship with the completely integrable system (1-1) and Sturm comparison techniques adapted to this special situation.

\section{The Schwarzian derivative operator}

For $\Omega \subset \mathbb{C}^{n}$ open, let $F: \Omega \rightarrow \mathbb{C}^{n}, F\left(z_{1}, \ldots, z_{n}\right)=\left(w_{1}, \ldots, w_{n}\right)$, be a locally univalent holomorphic mapping, and set $\Delta=\operatorname{det}(\partial F / \partial z)$. For $k=1, \ldots, n$, define an $n \times n$ matrix

$$
\mathbb{S}^{k} F=\left(S_{i j}^{k} F\right), \quad i, j=1, \ldots, n .
$$

Proposition 2.1. Let $F$ be a locally injective holomorphic mapping and let $w=$ $G(z)$ be a Möbius transformation. Then

$$
\mathbb{S}^{k}(F \circ G)=\sum_{r=1}^{n} \frac{\partial z_{k}}{\partial w_{r}} D G^{t}\left(\left(\mathbb{S}^{r} F\right) \circ G\right) D G \quad \text { for } k=1, \ldots, n .
$$


Proof. From (1-2) and the Möbius property of $G$ we have

$$
\begin{aligned}
S_{i j}^{k}(F \circ G)(z) & =\sum_{l, m, r=1}^{n} \mathbb{S}_{l m}^{r} F(w) \frac{\partial w_{l}}{\partial z_{i}} \frac{\partial w_{m}}{\partial z_{j}} \frac{\partial z_{k}}{\partial w_{r}}+S_{i j}^{k} G(z) \\
& =\sum_{r=1}^{n} \frac{\partial z_{k}}{\partial w_{r}} \sum_{m, l=1}^{n} \frac{\partial w_{l}}{\partial z_{i}} \mathbb{S}_{l m}^{r} F(w) \frac{\partial w_{m}}{\partial z_{j}}+S_{i j}^{k} G(z) \\
& =\sum_{r=1}^{n} \frac{\partial z_{k}}{\partial w_{r}} \sum_{m, l=1}^{n} \frac{\partial w_{l}}{\partial z_{i}} \mathbb{S}_{l m}^{r} F(w) \frac{\partial w_{m}}{\partial z_{j}} .
\end{aligned}
$$

The proposition follows after rewriting this in terms of matrices.

Definition 2.2. The Schwarzian derivative operator is the operator $\mathscr{Y} F(z): T_{z} \Omega \rightarrow$ $T_{F(z)} \Omega$ given by

$$
\mathscr{S} F(z)(\vec{v})=\left(\vec{v}^{t} \mathbb{S}^{1} F(z) \vec{v}, \vec{v}^{t} \mathbb{S}^{2} F(z) \vec{v}, \ldots, \vec{v}^{t} \mathbb{S}^{n} F(z) \vec{v}\right),
$$

where $\vec{v} \in T_{z} \Omega$.

Recall that the Bergman metric on $\mathbb{B}^{n}$ is the hermitian product defined by

$$
g_{i j}(z)=\frac{n+1}{\left(1-|z|^{2}\right)^{2}}\left(\left(1-|z|^{2}\right) \delta_{i j}+\bar{z}_{i} z_{j}\right) .
$$

Any automorphism of the ball is an isometry of the Bergman metric.

We define the norm of the Schwarzian derivative operator by

$$
\|\mathscr{S} F(z)\|=\sup _{\|\vec{v}\|=1}\|\mathscr{S} F(z)(\vec{v})\|,
$$

where $\|\vec{v}\|=\left(\sum g_{i j} v_{i} \bar{v}_{j}\right)^{1 / 2}$ is the Bergman norm of $\vec{v} \in T_{z} \mathbb{B}^{n}$.

A routine calculation using the fact that $u_{0}=\Delta^{-1 / n+1}$ is a solution of (1-1) with $P_{i j}^{k}=S_{i j}^{k} F$ allows one to rewrite the Schwarzian derivative operator as

$$
\mathscr{S} F(z)(\vec{v}, \vec{v})=(D F(z))^{-1} D^{2} F(z)(\vec{v}, \vec{v})-\frac{2}{n+1}\left(\frac{1}{\Delta} \sum_{j=1}^{n} \Delta_{j}(z) v_{j}\right) \vec{v},
$$

or yet

$$
\mathscr{S} F(z)(\vec{v}, \vec{v})=(D F(z))^{-1} D^{2} F(z)(\vec{v}, \vec{v})+2 \Delta^{1 / n+1}\left(\nabla u_{0} \cdot \vec{v}\right) \vec{v},
$$

where $\Delta_{j}=\sum_{k=1}^{n}(-1)^{j-1} \delta_{j k}$ and $\delta_{j k}$ is the determinant of $D F(z)$ with the $k$-th column replaced by the column

$$
\left(\frac{\partial^{2} f_{1}}{\partial z_{j} \partial z_{k}}, \ldots, \frac{\partial^{2} f_{n}}{\partial z_{j} \partial z_{k}}\right)^{\mathrm{tr}}(z)
$$


The operator $(D F(z))^{-1} D^{2} F(z)(z, \cdot)$ was considered by Pfaltzgraff [1974] in his generalization of the Becker criterion.

Theorem 2.3. Let $F: B^{n} \rightarrow \mathbb{C}^{n}$ be a locally injective holomorphic mapping and let $\sigma$ be an automorphism of $\mathbb{B}^{n}$. Then

$$
\|\mathscr{S}(F \circ \sigma)(z)\|=\|\mathscr{S}(\sigma(z))\| .
$$

Proof. We know that

$$
\begin{aligned}
\mathbb{S}^{k}(F \circ \sigma) & =\sum_{l=1}^{n} \frac{\partial z_{k}}{\partial w_{l}}(D \sigma)^{t} \mathbb{S}^{l} F \circ \sigma(D \sigma) \\
& =\left(\frac{\partial z_{k}}{\partial w_{1}}, \ldots, \frac{\partial z_{k}}{\partial w_{n}}\right)\left(\begin{array}{c}
(D \sigma)^{t} \mathbb{S}^{1} F \circ \sigma(D \sigma) \\
\vdots \\
(D \sigma)^{t} \mathbb{S}^{n} F \circ \sigma(D \sigma)
\end{array}\right) .
\end{aligned}
$$

Hence

$$
(\mathscr{S} F \circ \sigma)(z)(\vec{v})=D \sigma^{-1}\left(\begin{array}{c}
\vec{v}^{t}(D \sigma)^{t} \mathbb{S}^{1} F(\sigma(z))(D \sigma) \vec{v} \\
\vdots \\
\vec{v}^{t}(D \sigma)^{t} \mathbb{S}^{n} F(\sigma(z))(D \sigma) \vec{v}
\end{array}\right)=D \sigma^{-1}\left(\begin{array}{c}
\vec{u}^{t} \mathbb{S}^{1} F(\sigma(z)) \vec{u} \\
\vdots \\
\vec{u}^{t} \mathbb{S}^{n} F(\sigma(z)) \vec{u}
\end{array}\right),
$$

where $\vec{u}=D \sigma(z)(\vec{v})$. Then

$$
\|\mathscr{S}(F \circ \sigma)(z)(\vec{v})\|=\left\|D G \sigma^{-1} \mathscr{S} F(\sigma(z))(\vec{u})\right\|=\|\mathscr{S} F(\sigma(z))(\vec{u})\|,
$$

and since $\sigma$ is a isometry in the Bergman metric, the theorem follows after taking supremum over all unit vectors $\vec{v}$.

The definition of norm for the Schwarzian operator can be given using any hermitian metric or even a Finsler metric. Since in ball the Bergman metric coincides up to constant multiples with the Kobayashi or the Carathéodory metric, the resulting norm for $\mathscr{S F}$ is the same. This will certainly not be the case on arbitrary domains. Theorem 2.3 will also fail on arbitrary domains because it requires the automorphisms to be Möbius.

\section{The family $\mathscr{F}_{\alpha}$}

Definition 3.1. Consider the family

$$
L S=\left\{F: B^{n} \rightarrow \mathbb{C}^{n} \mid F(0)=0, D F(0)=\mathrm{Id}\right\}
$$

of normalized locally biholomorphic mappings on the ball $\mathbb{B}^{n}$, and the Koebe transformations $\Lambda_{\sigma}(F)$ of the ball, given by

$$
\Lambda_{\sigma}(F)(z)=(D \sigma(0))^{-1}(D F(\sigma(0)))^{-1}(F(\sigma(z))-F(\sigma(0)))
$$


for $F \in L S$ and $\sigma \in$ Aut $B^{n}$. A family $\mathscr{F} \subseteq L S$ is called linearly invariant (LIF) if $\Lambda_{\sigma}(F) \in \mathscr{F}_{F}$ for all $F \in \mathscr{F}_{F}$ and $\sigma \in$ Aut $B^{n}$.

This extends the notion of a linearly invariant family in one dimension, that is, a family $\mathscr{F}$ of analytic functions $f(z)=z+a_{2} z^{2}+\cdots$ defined on $\mathbb{D}$ that is closed under Koebe transformations

$$
g(z)=\frac{f\left(\frac{z_{0}+z}{1+\bar{z}_{0} z}\right)-f\left(z_{0}\right)}{\left(1-\left|z_{0}\right|^{2}\right) f^{\prime}\left(z_{0}\right)}, \quad z_{0} \in \mathbb{D} .
$$

In one dimension, several properties such as growth, covering, distortion and compactness are determined by the $\operatorname{order} \sup _{f \in \mathscr{F}} a_{2}(f)$ of the family $\mathscr{F}$. Pommerenke [1964] showed that the linearly invariant family defined by a Schwarzian derivative bound, $\mathscr{F}_{\alpha}=\left\{f: \mathbb{D} \rightarrow \mathbb{C}: f(0)=0, f^{\prime}(0)=1,\|S f\| \leq \alpha\right\}$, has norm $\sqrt{1+\alpha / 2}$.

Definition 3.2. The order of a linearly invariant family $\mathscr{F}$ in arbitrary dimension is defined as

$$
\operatorname{ord} \mathscr{F}=\sup _{F \in \mathscr{F}} \sup _{|\vec{v}|=1}\left|\operatorname{tr}\left\{\frac{1}{2} D^{2} F(0)(\vec{v}, \cdot)\right\}\right|,
$$

where $|\vec{v}|$ is the Euclidean norm of $\vec{v}$.

The order of an LIF $\mathscr{F}$ can be written equivalently as

$$
\operatorname{ord} \mathscr{F}=\sup _{F \in \mathscr{F}}\left|\frac{1}{2} \sum_{j=1}^{n} \frac{\partial^{2} f_{j}}{\partial z_{j} \partial z_{k}}(0)\right|
$$

(see [Pfaltzgraff 1997]). For example, for $n=2$ a straightforward computation shows that the order is

$$
\sup _{F \in \mathscr{F}}\left|\frac{1}{2} \frac{\partial^{2} f_{1}}{\partial z_{1}^{2}}(0,0)+\frac{\partial^{2} f_{2}}{\partial z_{1} \partial z_{2}}(0,0)\right| .
$$

Pfaltzgraff and Suffridge [2000] have introduced the notion of norm order, which has much broader applicability to the study of geometric properties of locally biholomorphic mappings than does the order. Consider the Taylor expansion

$$
F(z)=z+\frac{1}{2} D^{2} F(0)(z, z)+\cdots=z+A_{2}(z, z)+A_{3}(z, z, z)+\cdots,
$$

where $A_{m}(\cdot, \ldots, \cdot)=(1 / m !) D^{m} F(0)$, for $m=1,2, \ldots$, is an $m$-linear symmetric mapping. Then

$$
\left\|A_{m}\right\|=\sup _{|\lambda| \leq 1}\left|A_{m}(\lambda, \ldots, \lambda)\right| .
$$

Definition 3.3. The norm order of a linearly invariant family $\mathscr{F}$ is defined as

$$
\|\operatorname{Ord}\| \mathscr{F}=\sup _{F \in \mathscr{F}}\left\|A_{2}(F)\right\| .
$$


We define

$$
\mathscr{F}_{\alpha}=\left\{F: \mathbb{B}^{n} \rightarrow \mathbb{C}^{n} \mid F(0)=0, \quad D F(0)=\mathrm{Id},\|\mathscr{S} F(z)\| \leq \alpha\right\} .
$$

By Theorem 2.3, this is an LIF.

Remark 3.4. The task of calculating the exact value of the norm of $\mathscr{Y} F$ is, in general, not easy, especially because the Bergman and the Euclidean metrics are not conformal. For example, define a locally univalent holomorphic mapping in the ball $\mathbb{B}^{n}$ by $F_{\delta}=\left(f\left(z_{1}\right), \hat{z} g\left(z_{1}\right)\right)$, where $\hat{z}=\left(0, z_{2}, \ldots, z_{n}\right)$,

$$
g\left(z_{1}\right)=\frac{1}{1-z_{1}} \quad \text { and } \quad f\left(z_{1}\right)=\frac{1}{2 \delta}\left(\left(\frac{1+z_{1}}{1-z_{1}}\right)^{\delta}-1\right) .
$$

For $n=2$ a direct calculation shows that

$$
\mathbb{S}^{1} F_{\delta}(z)=\left(\begin{array}{cc}
\frac{2(\delta-1)}{3\left(1-z_{1}^{2}\right)} & 0 \\
0 & 0
\end{array}\right), \quad \mathbb{S}^{2} F_{\delta}(z)=\left(\begin{array}{cc}
\frac{2 z_{2}(1-\delta)}{\left(1-z_{1}\right)^{2}\left(1+z_{1}\right)} & -\frac{2(\delta-1)}{3\left(1-z_{1}^{2}\right)} \\
-\frac{2(\delta-1)}{3\left(1-z_{1}^{2}\right)} & 0
\end{array}\right) .
$$

Then

$$
\mathscr{S} F_{\delta}(z)(\vec{v})=\frac{2(\delta-1)}{3\left(1-z_{1}^{2}\right)}\left(v_{1}^{2}, \frac{-3 z_{2}}{1-z_{1}} v_{1}^{2}-2 v_{1} v_{2}\right) .
$$

Is easy to see that for $z_{2}=0$ the norm of the Schwarzian operator is

$$
\left\|\mathscr{S} F_{\delta}(z)\right\|=\frac{4}{9}(\delta-1), \quad \delta>1,
$$

while for $z_{1}=0$ with a little bit more effort one can show that

$$
\left\|\mathscr{S} F_{\delta}(z)\right\| \frac{2}{\sqrt{3}}(\delta-1), \quad \delta>1 .
$$

For arbitrary $z \in \mathbb{B}^{2}$ we had to resort to a numerical calculation in AMPL [Fourer et al. 2003]. The numerical results show that

$$
\left\|\mathscr{Y} F_{\delta}(z)\right\| \leq \frac{2}{\sqrt{3}}(\delta-1), \quad \delta>1 .
$$

On the other hand, Pfaltzgraff and Suffridge [2000] have shown that the norm order of the linear family generated for $F_{\delta}$ is equal to $\delta$; then for $\delta=\frac{\sqrt{3}}{2} \alpha+1$ the norm of Schwarzian operator of $F_{\delta}$ is $\alpha$, so that $F_{\delta} \in \mathscr{F}_{\alpha}$ and

$$
\|\operatorname{Ord}\| \mathscr{F}_{\alpha} \geq \frac{\sqrt{3}}{2} \alpha+1 .
$$

Pfaltzgraff and Suffridge [2000] show that an LIF is normal if and only if the norm order is bounded. Our aim is to study the family $\mathscr{F}_{\alpha}$, and we shall prove that it is normal. We begin with some lemmas. 
Lemma 3.5. Let $F$ be a holomorphic mapping in $\mathscr{F}_{\alpha}$. For $z_{1}=\left(z_{1}, 0, \ldots, 0\right) \in \mathbb{B}^{n}$,

$$
\begin{array}{ll}
\left|S_{11}^{1} F\left(z_{1}\right)\right| \leq \frac{\sqrt{n+1} \alpha}{1-\left|z_{1}\right|^{2}}, & \\
\left|S_{i i}^{1} F\left(z_{1}\right)\right| \leq \sqrt{n+1} \alpha & \text { for } i=2,3, \ldots, n, \\
\left|S_{11}^{k} F\left(z_{1}\right)\right| \leq \frac{\sqrt{n+1} \alpha}{\left(1-\left|z_{1}\right|^{2}\right)^{3 / 2}} & \text { for } k=2,3, \ldots, n, \\
\left|S_{1 j}^{k} F\left(z_{1}\right)\right| \leq \frac{2 \sqrt{n+1} \alpha}{1-\left|z_{1}\right|^{2}} & \text { for } k, j=2,3, \ldots, n, \\
\left|S_{1 j}^{1} F\left(z_{1}\right)\right| \leq \frac{2 \sqrt{n+1} \alpha}{\left(1-\left|z_{1}\right|^{2}\right)^{1 / 2}} & \text { for } j=2,3, \ldots, n, \\
\left|S_{i j}^{1} F\left(z_{1}\right)\right| \leq 2 \sqrt{n+1} \alpha & \text { for } i \neq j \neq 1, \\
\left|S_{i i}^{k} F\left(z_{1}\right)\right| \leq \frac{\sqrt{n+1} \alpha}{\left(1-\left|z_{1}\right|^{2}\right)^{1 / 2}} & \text { for } k, i=2,3, \ldots, n, \\
\left|S_{i j}^{k} F\left(z_{1}\right)\right| \leq \frac{2 \sqrt{n+1} \alpha}{\left(1-\left|z_{1}\right|^{2}\right)^{1 / 2}} & \text { for } k \neq 1, i \neq j \neq 1 .
\end{array}
$$

Proof. From (2-1) we have

$$
g_{11}\left(z_{1}, 0,0, \ldots, 0\right)=\frac{n+1}{\left(1-\left|z_{1}\right|^{2}\right)^{2}} \quad \text { and } \quad g_{i j}\left(z_{1}, 0,0, \ldots, 0\right)=\frac{n+1}{\left(1-\left|z_{1}\right|^{2}\right)},
$$

for all $i, j \neq 1$. Let $\vec{v}$ be a unit vector in the Bergman metric. Since $\left\|\mathscr{Y} F\left(z_{1}\right)(\vec{v})\right\| \leq$ $\alpha$, by setting $\vec{v}=(\lambda, 0, \ldots, 0)$ with $\lambda=\left(1-\left|z_{1}\right|^{2}\right) / \sqrt{n+1}$ we obtain $\left\|\mathscr{S} F\left(z_{1}, 0, \ldots, 0\right)(\vec{v})\right\|^{2}=(n+1)\left(\frac{\left|S_{11}^{1} \lambda^{2}\right|^{2}}{\left(1-\left|z_{1}\right|^{2}\right)^{2}}+\frac{\left|S_{11}^{2} \lambda^{2}\right|^{2}}{1-\left|z_{1}\right|^{2}}+\cdots+\frac{\left|S_{11}^{n} \lambda^{2}\right|^{2}}{1-\left|z_{1}\right|^{2}}\right) \leq \alpha^{2}$, whence (i) and (iii) follow. Now consider $\vec{v}=\left(0,0, \ldots, \lambda_{k}, 0, \ldots, 0\right)$ with $\lambda_{k}^{2}=$ $\left(1-\left|z_{1}\right|^{2}\right) /(n+1)$. As above we have that $\vec{v}$ is a unit vector in the Bergman metric. Since $\left\|\mathscr{Y} F\left(z_{1}, 0, \ldots, 0\right)(\vec{v})\right\| \leq \alpha$ then (ii) and (vii) follow. We obtain (vi) and (vii) analogously, by setting $\vec{v}=\left(0, \ldots, \lambda_{i}, 0, \ldots, \lambda_{j}, 0, \ldots, 0\right)$, where

$$
\lambda_{i}=\lambda_{j}=\frac{1}{\sqrt{2}} \frac{\left(1-\left|z_{1}\right|^{2}\right)^{1 / 2}}{\sqrt{n+1}} .
$$

Finally, (iv) and (v) are established by letting $\vec{v}=\left(\lambda_{1}, \ldots, \lambda_{j}, 0, \ldots, 0\right)$, with

$$
\lambda_{1}=\frac{1}{\sqrt{2}} \frac{\left(1-\left|z_{1}\right|^{2}\right)}{\sqrt{n+1}} \quad \text { and } \quad \lambda_{2}=\frac{1}{\sqrt{2}} \frac{\left(1-\left|z_{1}\right|^{2}\right)^{1 / 2}}{\sqrt{n+1}} .
$$


Lemma 3.6. If $F \in \mathscr{F}_{\alpha}$ we have

$$
\left|S_{11}^{0} F\left(z_{1}, 0, \ldots, 0\right)\right| \leq \frac{C(n, \alpha)}{\left(1-\left|z_{1}\right|^{2}\right)^{2}}
$$

with

$$
C(n, \alpha)=\left(4 n^{2}+2 n-2+\frac{n+1}{n-1}\right) \alpha^{2}+\left(4 \sqrt{n+1}+8 \frac{\sqrt{n+1}}{n-1}\right) \alpha,
$$

and

$$
\left|S_{1 j}^{0} F\left(z_{1}, 0, \ldots, 0\right)\right| \leq \frac{K(n, \alpha)}{\left(1-\left|z_{1}\right|^{2}\right)^{3 / 2}},
$$

with

$$
K(n, \alpha)=(16+3 \sqrt{2}) \sqrt{n+1} \alpha+6\left(n^{2}-1\right) \alpha .
$$

Proof. Differentiating (1-1) and using Proposition 1.2 we get

$$
\begin{aligned}
& S_{i i}^{0} F(z)=-\frac{1}{n-1} \sum_{k=1}^{n} \frac{\partial}{\partial z_{k}} S_{i i}^{k} F(z)+\frac{1}{n-1} \sum_{k=1}^{n} \sum_{j=1}^{n} S_{i j}^{k} F(z) S_{k i}^{j} F(z), \\
& S_{i j}^{0} F(z)=\frac{\partial}{\partial z_{j}} S_{i i}^{i} F(z)-\frac{\partial}{\partial z_{i}} S_{i j}^{i} F(z)+\sum_{k=1}^{n} S_{i i}^{k} F(z) S_{k j}^{i} F(z)-\sum_{k=1}^{n} S_{i j}^{k} F(z) S_{k i}^{i} F(z)
\end{aligned}
$$

for $i \neq j$. Thus, the coefficients $S_{i j}^{0}$ depend on the $S_{i j}^{k}$. Let $F\left(z_{1}\right)=F\left(z_{1}, 0, \ldots, 0\right)$, so that for all mappings in $\mathscr{F}_{\alpha}$ we have

$$
\begin{aligned}
&\left|S_{11}^{0} F\left(z_{1}\right)\right| \leq \frac{1}{n-1} \sum_{k=1}^{n}\left|\frac{\partial}{\partial z_{k}} S_{11}^{k} F\left(z_{1}\right)\right|+\frac{1}{n-1} \sum_{k=1}^{n} \sum_{j=1}^{n}\left|S_{1 j}^{k} F\left(z_{1}\right)\right|\left|S_{k 1}^{j} F\left(z_{1}\right)\right|, \\
&=\frac{1}{n-1} \sum_{k=1}^{n}\left|\frac{\partial}{\partial z_{k}} S_{11}^{k} F\left(z_{1}\right)\right|+\frac{1}{n-1} \sum_{k=2}^{n} \sum_{j=2}^{n}\left|S_{1 j}^{k} F\left(z_{1}\right)\right|\left|S_{k 1}^{j} F\left(z_{1}\right)\right| \\
&+\frac{1}{n-1} \sum_{k=2}^{n}\left|S_{11}^{k} F\left(z_{1}\right)\right|\left|S_{k 1}^{1} F\left(z_{1}\right)\right|+\frac{1}{n-1}\left|S_{11}^{1} F\left(z_{1}\right)\right|^{2} .
\end{aligned}
$$

Therefore Lemma 3.5 implies

$$
\begin{aligned}
& \left|S_{11}^{0} F\left(z_{1}, 0, \ldots, 0\right)\right| \\
& \quad \leq \frac{4(n+1)(n-1) \alpha^{2}}{\left(1-\left|z_{1}\right|^{2}\right)^{2}}+\frac{2(n+1) \alpha^{2}}{\left(1-\left|z_{1}\right|^{2}\right)^{2}}+\frac{\frac{n+1}{n-1} \alpha^{2}}{\left(1-\left|z_{1}\right|^{2}\right)^{2}}+\frac{1}{n-1} \sum_{k=1}^{n}\left|\frac{\partial}{\partial z_{k}} S_{11}^{k} F\left(z_{1}\right)\right| .
\end{aligned}
$$

Since $F \in \mathscr{F}_{\alpha}$, by taking the unit vector $\vec{v}=(\lambda, 0, \ldots, 0)$ where

$$
|\lambda|^{2}=\frac{\left(1-\left|z_{1}\right|^{2}-\left|z_{k}\right|^{2}\right)^{2}}{(n+1)\left(1-\left|z_{k}\right|^{2}\right)}
$$


in the Bergman metric, a straightforward calculation shows that

$$
\left|S_{11}^{k} F\left(z_{1}, 0, \ldots 0, z_{k}, 0, \ldots, 0\right)\right| \leq \frac{\sqrt{n+1} \alpha\left(1-\left|z_{k}\right|^{2}\right)}{\left(1-\left|z_{1}\right|^{2}-\left|z_{k}\right|^{2}\right)^{3 / 2}} \quad \text { for } k \neq 1 .
$$

By considering $S_{11}^{k} F\left(z_{1}, 0, \ldots 0, z_{k}, 0, \ldots, 0\right)$ as a holomorphic function of $z_{k}$ we deduce from Cauchy's integral formula that

$$
\left|\frac{\partial}{\partial z_{k}} S_{11}^{k} F\left(z_{1}, 0, \ldots, 0\right)\right| \leq \frac{4 \sqrt{n+1} \alpha}{\left(1-\left|z_{1}\right|^{2}\right)^{2}} \quad \text { for } k \neq 1 .
$$

Similarly,

$$
\left|\frac{\partial}{\partial z_{1}} S_{11}^{1} F\left(z_{1}, 0, \ldots, 0\right)\right| \leq \frac{8 \sqrt{n+1} \alpha}{\left(1-\left|z_{1}\right|^{2}\right)^{2}} .
$$

Using these two inequalities we conclude that

$$
\begin{aligned}
& \left|S_{11}^{0} F\left(z_{1}, 0, \ldots, 0\right)\right| \\
& \quad \leq \frac{\left(4 n^{2}+2 n-2\right) \alpha^{2}}{\left(1-\left|z_{1}\right|^{2}\right)^{2}}+\frac{\frac{n+1}{n-1} \alpha^{2}}{\left(1-\left|z_{1}\right|^{2}\right)^{2}}+\frac{4 \sqrt{n+1} \alpha}{\left(1-\left|z_{1}\right|^{2}\right)^{2}}+\frac{1}{n-1} \frac{8 \sqrt{n+1} \alpha}{\left(1-\left|z_{1}\right|^{2}\right)^{2}} .
\end{aligned}
$$

For $j \neq 1$ we have

$$
\begin{aligned}
\left|S_{1 j}^{0} F\left(z_{1}\right)\right| \leq\left|\frac{\partial}{\partial z_{j}} S_{11}^{1} F\left(z_{1}\right)\right|+ & \left|\frac{\partial}{\partial z_{1}} S_{1 j}^{1} F\left(z_{1}\right)\right| \\
& \sum_{k=1}^{n}\left|S_{11}^{k} F\left(z_{1}\right)\right|\left|S_{k j}^{1} F\left(z_{1}\right)\right|+\left|S_{1 j}^{k} F\left(z_{1}\right)\right|\left|S_{k 1}^{1} F\left(z_{1}\right)\right|,
\end{aligned}
$$

The contribution of the last two summands is at most

$$
\frac{2 \alpha(n+1)(n-1)}{\left(1-\left|z_{1}\right|^{2}\right)^{3 / 2}}+\frac{4 \alpha(n+1)(n-1)}{\left(1-\left|z_{1}\right|^{2}\right)^{3 / 2}},
$$

while the first two can be estimated using Cauchy's integral formula:

$$
\left|\frac{\partial}{\partial z_{1}} S_{1 j}^{1} F\left(z_{1}\right)\right| \leq \frac{16 \sqrt{n+1} \alpha}{\left(1-\left|z_{1}\right|^{2}\right)^{3 / 2}}, \quad\left|\frac{\partial}{\partial z_{j}} S_{11}^{1} F\left(z_{1}\right)\right| \leq \frac{3 \sqrt{2} \sqrt{n+1} \alpha}{\left(1-\left|z_{1}\right|^{2}\right)^{3 / 2}} .
$$

Putting it all together,

$$
\left|S_{1 j}^{0} F\left(z_{1}\right)\right| \leq \frac{6 \alpha\left(n^{2}-1\right)}{\left(1-\left|z_{1}\right|^{2}\right)^{3 / 2}}+\frac{16 \sqrt{n+1} \alpha}{\left(1-\left|z_{1}\right|^{2}\right)^{3 / 2}}+\frac{3 \sqrt{2} \sqrt{n+1} \alpha}{\left(1-\left|z_{1}\right|^{2}\right)^{3 / 2}},
$$

proving the theorem. 
It is clear that if $u\left(z_{1}, \ldots, z_{n}\right)$ is a solution of the system (1-1) then $u\left(z_{1}\right)=$ $u\left(z_{1}, 0, \ldots, 0\right)$ satisfies

$$
u^{\prime \prime}=S_{11}^{1} u^{\prime}+\sum_{j=2}^{n} S_{11}^{j} \phi_{j}+S_{11}^{0} u \quad \text { and } \quad \phi_{k}^{\prime}=S_{1 k}^{1} u^{\prime}+\sum_{j=2}^{n} S_{1 k}^{j} \phi_{j}+S_{1 k}^{0} u
$$

for $k=2,3, \ldots, n$, where $\phi_{k}(z)=\partial u / \partial z_{k}$.

Lemma 3.7. Let $P=P(x), Q=Q(x)$ be continuous functions defined on $[0,1)$, with $Q(x) \geq 0$. Let $u=u(x), v=v(x)$ satisfy

$$
\begin{aligned}
& u^{\prime \prime}+P u+Q \geq 0, u(0)=1, u^{\prime}(0)=0, \\
& v^{\prime \prime}+P v+Q=0, v(0)=1, v^{\prime}(0)=0 .
\end{aligned}
$$

Then $u \geq v$ on $\left[0, x_{0}\right)$, where $x_{0}$ is the first zero of $v$.

Proof. For $\varepsilon>0$, let $u_{\varepsilon}=u+\varepsilon y$, where $y$ is solution of $y^{\prime \prime}+P y=0, y(0)=0$, $y^{\prime}(0)=1$. Then $w=u_{\varepsilon}^{\prime} v-v^{\prime} u_{\varepsilon}$ satisfies $w(0)=\varepsilon>0$ and $w^{\prime} \geq Q\left(u_{\varepsilon}-v\right)$. Because of the initial conditions of $u_{\varepsilon}$ and $v$, the function $w$ has $w^{\prime}>0$ on an interval $(0, r)$. But then $w>0$ (in fact, $\geq \varepsilon$ ) on that interval, which implies that $u_{\varepsilon}^{\prime} / u_{\varepsilon}>v^{\prime} / v$ if $v>0$, thus $u_{\varepsilon}>v$. It follows from this argument that the first zero of $u_{\varepsilon}$ cannot occur before the first zero of $v$, and the lemma obtains after letting $\varepsilon \rightarrow 0$.

Lemma 3.8. Let $u$ be a solution of the system (1-1) satisfying $u(0, \ldots, 0)=1$, $\nabla u(0, \ldots, 0)=0$ and $P_{i j}^{k}=S_{i j}^{k} F$ with $F \in \mathscr{F}_{\alpha}$. Then there exists $r>0$ and $\delta>0$ such that $|u|>\delta>0$ for $|z|<r$.

Proof. Let $z_{0} \in \mathbb{B}^{n}$ be a zero of $u$ of smallest euclidean norm, that is, $u\left(z_{0}\right)=$ 0 and $u(z) \neq 0$ for $|z|<\left|z_{0}\right|=r_{0}$. Since $\mathscr{F}_{\alpha}$ is a linearly invariant family we can assume that $z_{0}=\left(x_{0}, 0, \ldots, 0\right)$. We shall study the zeros of the function $u(x)=u(x, 0, \ldots, 0)$ in $0<x<1$. If $F(x)=F(x, 0, \ldots, 0)$, then $u(x)$ and $\varphi_{k}(x)=\left(\partial u / \partial z_{k}\right)(x, 0, \ldots, 0)$ satisfies the system

$$
\begin{aligned}
& u^{\prime \prime}(x)=\sum_{k=1}^{n} S_{11}^{k} F(x) \varphi_{k}(x)+S_{11}^{0} F(x) u(x), \\
& \varphi_{j}^{\prime}(x)=\sum_{k=1}^{n} S_{1 j}^{k} F(x) \varphi_{k}(x)+S_{1 j}^{0} F(x) u(x), \quad j=2, \ldots, n,
\end{aligned}
$$

with initial conditions $u(0)=1$ and $\varphi_{k}(0)=0$. With $\theta=\left(\varphi_{1}, \ldots, \varphi_{n}, u\right)$, we can rewrite the system (3-3) as

$$
\theta^{\prime}(x)=A(x) \cdot \theta(x), \quad \theta(0)=(0,0, \ldots, 1),
$$

where $A(x)$ is the $(n+1) \times(n+1)$ matrix of coefficients of the system. Let $f^{2}(x)=$ $\|\theta(x)\|^{2}$ be the square of the Euclidean norm of $\theta(x)$. Using - to represent the 
Euclidean inner product of vectors in $\mathbb{C}^{n+1}=\mathbb{R}^{2 n+2}$, we have

$$
f^{\prime}(x) f(x)=\theta^{\prime}(x) \cdot \theta(x)=A(x) \theta(x) \cdot \theta(x) ;
$$

therefore $f^{\prime}(x) f(x) \leq\|A(x)\|\|\theta(x)\|^{2}=\|A(x)\| f^{2}(x)$, so

$$
\frac{f^{\prime}(x)}{f(x)} \leq\|A(x)\| \text {. }
$$

Since $f(0)=1$ we conclude that $f(x) \leq e^{\int_{0}^{x} p(s) d s}$, where $p(s)$ stands for the bounds obtained for $\|A(s)\|$ from Lemmas 3.5 and 3.6. In particular, we have

$$
\left|u^{\prime}(x)\right| \leq e^{\int_{0}^{x} p(s) d s}, \quad\left|\varphi_{k}(x)\right| \leq e^{\int_{0}^{x} p(s) d s} \quad \text { for } k=2, \ldots, n .
$$

Setting $U^{2}(x)=|u(x)|^{2}$, we obtain $2 U U^{\prime}=2 \operatorname{Re}\left(u^{\prime} \bar{u}\right)$, hence $\left(U^{\prime}\right)^{2}+U U^{\prime \prime}=$ $\operatorname{Re}\left(u^{\prime \prime} \bar{u}\right)+\left|u^{\prime}\right|^{2}, U(0)=1, U^{\prime}(0)=0$. Since $\left|U^{\prime}\right| \leq\left|u^{\prime}\right|$, we have

$$
U U^{\prime \prime} \geq \operatorname{Re}\left(u^{\prime \prime} \bar{u}\right) \text {. }
$$

Using this in (3-3) we get

$$
U U^{\prime \prime} \geq \operatorname{Re}\left\{S_{11}^{0} F(x)\right\} U^{2}+\operatorname{Re}(q(x) \bar{u}),
$$

where $q(x)=S_{11}^{1} F(x) u^{\prime}(x)+\sum_{k=2}^{n} S_{11}^{k} F(x) \varphi_{k}(x)$; hence

$$
U^{\prime \prime} \geq-\left|S_{11}^{0} F(x)\right| U-|q(x)|,
$$

or $U^{\prime \prime}+P(x) U+Q(x) \geq 0$, where $P$ and $Q$ are the bounds obtained from Lemmas 3.5 and 3.6 for $\left|S_{11}^{0} F(x, 0, \ldots, 0)\right|$ and $|q(x)|$, respectively. It follows now from Lemma 3.7 that $U \geq v$ on $\left[0, x_{0}\right)$, where $x_{0}$ is the first zero of $v$, which is solution of $v^{\prime \prime}+P v+Q=0, v(0)=1, v^{\prime}(0)=0$. The lemma follows taking $r<x_{0}$.

Remark 3.9. It is clear that we need to estimate the first zero of the function $v$. In fact, we proved that $\left|S_{11}^{0} F(x, 0, \ldots, 0)\right| \leq c(n, \alpha)\left(1-x^{2}\right)^{-2}=P$, where $c=c(n, \alpha)$ is a constant. Also one can obtain from Lemmas 3.5 and 3.6 a bound of $|q(x)|$ of the form

$$
|q(x)| \leq \frac{M}{\left(1-x^{2}\right)^{\delta+1}}=Q,
$$

where $M=\sqrt{n(n+1)} \alpha$ and $\delta$ also depends on $n$ and $\alpha$. Then $v$ is a solution of

$$
v^{\prime \prime}+\frac{c}{\left(1-x^{2}\right)^{2}} v+\frac{M}{\left(1-x^{2}\right)^{\delta+1}}=0, \quad v(0)=1, \quad v^{\prime}(0)=0 .
$$

In general, for given constants $c, M, \delta$, one will be able to estimate the first zero of $v$ only numerically. However, if $\delta<1$ then by comparison, it follows that the first zero of $v$ does not occur before the first zero of the solution $w$ of

$$
w^{\prime \prime}+\frac{c}{\left(1-x^{2}\right)^{2}} w+\frac{M}{\left(1-x^{2}\right)^{2}}=0, \quad w(0)=1, \quad w^{\prime}(0)=0,
$$


and this can be determined analytically. Indeed we have $w=(M+1) y_{c}-M$, where $y_{c}$ is the solution of

$$
y^{\prime \prime}+\frac{c}{\left(1-x^{2}\right)^{2}} y=0, \quad y(0)=1, \quad y^{\prime}(0)=0,
$$

which can be found, for example, in [Kamke 1930]. Thus the first zero of $w$ is the solution of the (transcendental) equation $y_{c}(x)=M /(1+M)$.

Theorem 3.10. Fix $\alpha<\infty$. The family

$$
\mathscr{F}_{\alpha}=\left\{F: \mathbb{B}^{n} \rightarrow \mathbb{C}^{n} \mid F(0)=0, D F(0)=\mathrm{Id},\|\mathscr{Y} F(z)\| \leq \alpha\right\}
$$

is a normal family.

Proof. Let $F \in \mathscr{F}_{\alpha}$. From Proposition 1.2 we have

$$
F=\left(\frac{u_{1}}{u_{0}}, \ldots, \frac{u_{n}}{u_{0}}\right)=\left(f_{1}, \ldots, f_{n}\right),
$$

where $u_{i}$ and $u_{0}=\Delta^{-1 / n+1}$ are linearly independent solutions of (1-1) such that $\left(\partial u_{i} / \partial z_{k}\right)(0)=0$ for all $k \neq i$ and $\left(\partial u_{i} / \partial z_{i}\right)(0)=1$ for $i=1, \ldots, n$; see [Yoshida 1984]. From equation (2-2) we deduce that

$$
D^{2} F(0)(\vec{v}, \vec{v})=\mathscr{Y} F(0)(\vec{v}, \vec{v})+2\left(\nabla u_{0}(0) \cdot \vec{v}\right) \vec{v} .
$$

Hence $\left|A_{2}(z)\right|$ will be uniformly bounded for $F$ in the family $\mathscr{F}_{\alpha}$ provided that the same holds for the derivatives $\left|\left(\partial u_{0} / \partial z_{j}\right)(0)\right|$ for $j=1, \ldots, n$. To show the latter, consider the composition $G=T \circ F$ with the Möbius transformation given by

$$
T(z)=\frac{z}{1+z \cdot \bar{a}},
$$

where we have introduced the inner product $\langle z, w\rangle=z_{1} \bar{w}_{1}+\cdots+z_{n} \bar{w}_{n}$. Using (3-5), we get

$$
\begin{aligned}
G(z)=\frac{F(z)}{1+\langle F(z), \bar{a}\rangle} & =\left(\frac{u_{1}}{u_{0}+a_{1} u_{1}+\cdots+a_{n} u_{n}}, \ldots, \frac{u_{n}}{u_{0}+a_{1} u_{1}+\cdots+a_{n} u_{n}}\right) \\
& =\left(\frac{\tilde{u}_{1}}{\tilde{u}_{0}}, \ldots, \frac{\tilde{u}_{n}}{\tilde{u}_{0}}\right),
\end{aligned}
$$

where $\tilde{u}_{0}=u_{0}+a_{1} u_{1}+\cdots+a_{n} u_{n}$ and $\tilde{u}_{i}=u_{i}$ for $i=1, \ldots, n$. Differentiating and setting $a_{k}=\left(\partial u_{0} / \partial z_{k}\right)(0)$ for $k=1, \ldots, n$, we obtain $\nabla\left(\tilde{u}_{0}\right)(0)=0$. This may introduce a pole of $G$ but away from the origin. The function $\tilde{u}_{0}$ satisfies the system

$$
\frac{\partial^{2} \tilde{u}_{0}}{\partial z_{i} \partial z_{j}}(z)=\sum_{k=1}^{n} S_{i j}^{k} F(z) \frac{\partial \tilde{u}_{0}}{\partial z_{k}}+S_{i j}^{0} F(z) \tilde{u}_{0}(z), \quad \tilde{u}_{0}(0)=1, \quad \nabla \tilde{u}_{0}(0)=0,
$$


and in view of Lemma 3.8, $\tilde{u}_{0}$ does not vanish on $B_{r}$ for some $r>0$. At the same time, since satisfies $\tilde{u}_{i}(0)=0$ and $\left|\nabla \tilde{u}_{i}(0)\right|=1$ for each $i=1, \ldots, n$, it is easy to see from (1-1) and the bounds in Lemmas 3.5 and 3.6 that the functions $\tilde{u}_{i}$ will be uniformly bounded on compact subsets. Therefore, the class of mappings $G$ obtained with this normalization is normal on $|z|<r_{0}$ with $r_{0}<r$; then there exists $s_{0}>0$ such that $G\left(\mathbb{B}_{r_{0}}^{n}\right) \supset \mathbb{B}_{s_{0}}^{n}$. Since the image of $G:=\left(\tilde{f}_{1}, \ldots, \tilde{f}_{n}\right)$ covers a ball of radius $s_{0}$ and

$$
F=\frac{G}{1-\langle a, \tilde{f}\rangle}
$$

is holomorphic, we conclude that $\left|a_{1}\right|^{2}+\cdots+\left|a_{n}\right|^{2} \leq 1 / s_{0}^{2}$. This shows that $\left|\nabla u_{0}(0)\right|=\sqrt{\left|a_{1}\right|^{2}+\cdots+\left|a_{n}\right|^{2}}$ is uniformly bounded and the theorem follows.

In analogy to the result of Pommerenke cited on page 206, we have:

Theorem 3.11. $\|\operatorname{Ord}\| \mathscr{F}_{\alpha} \leq \frac{\sqrt{n+1}}{2} \alpha+\lambda_{\alpha}$, where $\lambda_{\alpha}=\frac{2 \sqrt{n}}{n+1}$ ord $\mathscr{F}_{\alpha}$.

Proof. Equation (2-2) yields $D^{2} F(0)(\vec{v}, \vec{v})=\mathscr{S} F(0)(\vec{v}, \vec{v})+2\left(\nabla u_{0}(0) \cdot \vec{v}\right) \vec{v}$. Is not difficult to see that

$$
\frac{\partial u_{0}}{\partial z_{k}}(0)=-\frac{1}{n+1} \sum_{j=1}^{n} \frac{\partial^{2} f_{j}}{\partial z_{j} \partial z_{k}}(0) ;
$$

hence, taking the Euclidean norm and the supremum over all unit vectors $\vec{v}$, we obtain

$$
\left|A_{2}(F)\right| \leq \frac{\sqrt{n+1}}{2}\|\mathscr{S} F(0)\|+\left|\nabla u_{0}(0)\right|,
$$

where $\|\cdot\|$ is the Bergman metric. Therefore

$$
\|\operatorname{Ord}\| F_{\alpha} \leq \frac{\sqrt{n+1}}{2} \alpha+\lambda_{\alpha} .
$$

Nehari [1949] proved that if $f$ belongs to the univalent class in the unit disk, the Schwarzian derivative of $f$ has norm at most 6; but this has no counterpart in higher dimensions, since the norm order of univalent mappings is infinite.

Corollary 3.12. Let $F$ be a convex holomorphic mapping in $\mathbb{B}^{2}$, then

$$
\|\mathscr{S} F(z)\| \leq \alpha_{K}, \quad \text { where } \alpha_{K}=\frac{2}{\sqrt{3}}+\frac{4 \sqrt{2}}{3 \sqrt{3}} \cdot 1.761 .
$$

Proof. Barnard, FitzGerald and Gong [Barnard et al. 1994] established that $\frac{3}{2} \leq$ ord $K\left(\mathbb{B}^{2}\right) \leq 1.761$ for the family of convex mappings $K\left(\mathbb{B}^{2}\right)$. Using (2-2) and setting the Bergman norm in the origin, we deduce that

$$
\|\mathscr{S} F(0)(\vec{v})\| \leq \sqrt{3}\left|D^{2} F(0)(\vec{v})\right|+2\left|\nabla u_{0}(0) \cdot \vec{v}\right|,
$$


where $|\cdot|$ is the Euclidean norm. Thus, taking the supremum over all vectors with $\|\vec{v}\|=1$, we obtain

$$
\|\mathscr{P} F(0)\| \leq \frac{2}{\sqrt{3}}\|\operatorname{Ord}\| K\left(\mathbb{B}^{2}\right)+\frac{4 \sqrt{2}}{3 \sqrt{3}} \operatorname{ord} K\left(\mathbb{B}^{2}\right) \leq \frac{2}{\sqrt{3}}+\frac{4 \sqrt{2}}{3 \sqrt{3}} \cdot 1.761 .
$$

To establish the estimate at an arbitrary point in the ball, apply the appropriate Koebe transform and Theorem 2.3.

The order of $K\left(\mathbb{B}^{n}\right)$ for $n \geq 2$ is unknown, but Liu [1989] has established an upper bound in any dimension. The conjecture in [Barnard et al. 1994] that ord $K\left(\mathbb{B}^{n}\right)=\frac{1}{2}(n+1)$ for $n \geq 2$ was shown to be false by Pfaltzgraff and Suffridge [2000].

Definition 3.13. A holomorphic mapping $F \in \mathscr{F}_{\alpha}$ is an extremal order function for $\mathscr{F}_{\alpha}$ if its order is equal to the order of family $\mathscr{F}_{\alpha}$.

Theorem 3.14. Let $F$ be a extremal order function for the family $\mathscr{F}_{\alpha}$. There exists $\left\{z_{k}\right\} \in \mathbb{B}^{n}$ with $\left|z_{k}\right| \rightarrow 1$ when $k \rightarrow \infty$, such that

$$
\lim _{k \rightarrow \infty}\left|F\left(z_{k}\right)\right|=\infty
$$

Proof. Let $F=\left(f_{1}, \ldots, f_{n}\right)=\left(u_{1} / u_{0}, \ldots, u_{n} / u_{0}\right)$ be an extremal order mapping and consider the Möbius transformation

$$
G=\left(\frac{f_{1}}{1+\varepsilon f_{1}}, \ldots, \frac{f_{n}}{1+\varepsilon f_{1}}\right),
$$

for $\varepsilon>0$. We have $\mathscr{S} F(z)=\mathscr{S} G(z), G(0)=0, D G(0)=\mathrm{Id}$ and we can write $G=\left(u_{1} / \tilde{u}_{0}, \ldots, u_{n} / \tilde{u}_{0}\right)$, where $\tilde{u_{0}}=u_{0}+\varepsilon u_{1}$. Differentiating with respect to $z_{1}$ and evaluating in the origin, we obtain

$$
\frac{\partial \tilde{u}_{0}}{\partial z_{1}}(0)=\frac{\partial u_{0}}{\partial z_{1}}(0)+\varepsilon .
$$

But is easy to see that

$$
\frac{\partial u_{0}}{\partial z_{1}}(0)=\frac{1}{n+1} \sum_{j=1}^{n} \frac{\partial^{2} f_{j}}{\partial z_{1} \partial z_{j}}(0)=\frac{2}{n+1} \text { ord } \mathscr{F}_{\alpha} .
$$

If $G$ were holomorphic in the ball, it would lie in $\mathscr{F}_{\alpha}$, contradicting the fact that $F$ is an extremal order function. Hence there must exist a point $z_{\varepsilon}$ such that $1+\varepsilon f_{1}\left(z_{\varepsilon}\right)=$ 0 , that is, $f_{1}\left(z_{\varepsilon}\right)=-1 / \varepsilon$. It is also clear that $\left|z_{\varepsilon}\right| \rightarrow 1$ when $\varepsilon \rightarrow 0$, which finishes the proof. 


\section{An estimate for $\lambda_{\alpha}$}

To find explicit bounds for $\lambda_{\alpha}$ in terms of $\alpha$ we have to estimate the radius $s_{0}$ of a ball covered by the function $G=\left(u_{1} / u_{0}, \ldots, u_{n} / u_{0}\right)$ considered in the proof of Theorem 3.10. Recall that the $u_{i}$ formed a set of linearly independent solutions of (1-1) with initial conditions $u_{0}(0)=1, \nabla u_{0}(0)=0, u_{i}(0)=0$ and $\left|\nabla u_{i}(0)\right|=1$ for $i=1, \ldots, n$. Set $u(x)=u_{k}(x, 0, \ldots, 0)$ and

$$
\theta(x)=\left(\frac{\partial u}{\partial z_{1}}(x), \frac{\partial u}{\partial z_{2}}(x)\left(1-x^{2}\right)^{-\frac{1}{2}}, \ldots, \frac{\partial u}{\partial z_{n}}(x)\left(1-x^{2}\right)^{-\frac{1}{2}}, u(x)\left(1-x^{2}\right)^{-1}\right) .
$$

It follows from Lemmas 3.5 and 3.6 that $\theta^{\prime}=B \theta$ for some modification $B$ of the matrix $A$ of (3-4), such that

$$
\|B(x)\| \leq \frac{k}{1-x^{2}} \quad \text { with } k=\delta(n, \alpha)+2,
$$

where $\delta(n, \alpha) \rightarrow 0$ when $\alpha \rightarrow 0$. As in the proof of Lemma 3.8 we obtain

$$
\|\theta(x)\| \leq\left(\frac{1+x}{1-x}\right)^{k / 2} .
$$

In particular, taking $u=u_{0}$ we get $\left|u_{0}(x)\right| \leq\left(1-x^{2}\right)\left(\frac{1+x}{1-x}\right)^{k / 2}$. Now we need to find a lower bound for $\left|u_{i}\right|, i=1, \ldots, n$. Consider the real function $U(x)=|u(x)|$, for which

$$
U^{\prime \prime} \geq-\left|S_{11}^{1} G(x) \frac{\partial u}{\partial z_{1}}(x)+\cdots+S_{11}^{n} G(x) \frac{\partial u}{\partial z_{n}}(x)+S_{11}^{0} G(x) u(x)\right| .
$$

Using (4-1) and Lemmas 3.5 and 3.6, we obtain

$$
U^{\prime \prime}+\frac{C}{\left(1-x^{2}\right)^{2}} U \geq-\frac{\sqrt{n(n+1)} \alpha}{1-x^{2}}\left(\frac{1+x}{1-x}\right)^{k / 2}, \quad U(0)=0, \quad U^{\prime}(0)=1 .
$$

Then $U \geq y$ until the first zero $x=x_{\alpha}$ of the solution $y$ of

$$
y^{\prime \prime}+\frac{C}{\left(1-x^{2}\right)^{2}} y=-\frac{\sqrt{n(n+1)} \alpha}{1-x^{2}}\left(\frac{1+x}{1-x}\right)^{k / 2}, \quad y(0)=0, \quad y^{\prime}(0)=1 .
$$

Hence

$$
|G(x)| \geq \frac{\sqrt{n} y(x)}{\left(1-x^{2}\right)\left(\frac{1+x}{1-x}\right)^{k / 2}}=\phi(x) .
$$

It follows that $G\left(\mathbb{B}_{x_{\alpha}}\right)$ covers a ball of radius $M_{\alpha}=\max \left\{\phi(x): 0<x \leq x_{\alpha}\right\}$. From the proof of Theorem 3.10 we finally see that

$$
\lambda_{\alpha} \leq \frac{1}{M_{\alpha}}
$$




\section{Acknowledgment}

We thank the referee for useful suggestions and an interesting discussion.

\section{References}

[Barnard et al. 1994] R. W. Barnard, C. H. FitzGerald, and S. Gong, "A distortion theorem for biholomorphic mappings in $\mathbb{C}^{2}$, Trans. Amer. Math. Soc. 344:2 (1994), 907-924. MR 94k:32034 Zbl 0814.32004

[Chuaqui and Osgood 1993] M. Chuaqui and B. Osgood, "Sharp distortion theorems associated with the Schwarzian derivative", J. London Math. Soc. (2) 48:2 (1993), 289-298. MR 94g:30005 Zbl 0792.30013

[Epstein 1986] C. L. Epstein, "The hyperbolic Gauss map and quasiconformal reflections", J. Reine Angew. Math. 372 (1986), 96-135. MR 88b:30029 Zbl 0591.30018

[Fourer et al. 2003] R. Fourer, D. M. Gay, and B. W. Kernighan, AMPL: a modeling language for mathematical programming, 2nd ed., Duxbury Press and Brooks/Cole, 2003.

[Kamke 1930] E. Kamke, Differentialgleichungen reeller Funktionen, Akademische Verlagsgesellschaft, Leipzig, 1930. Reprinted Chelsea, New York, 1948. JFM 56.0375.03

[Kraus 1932] W. Kraus, "Ueber den Zusammenhang einiger Charakteristiken eines einfach zusammenhangenden bereiches mit der Kreisabbildung”, Mitt. Math. Sem. Giessen 21 (1932), 1-28. Zbl 0005.30104

[Liu 1989] T. Liu, “The distortion theorem for biholomorphic mappings in $\mathbb{C}^{n}$ ”, Preprint, 1989.

[Molzon and Pinney Mortensen 1997] R. Molzon and K. Pinney Mortensen, "Univalence of holomorphic mappings", Pacific J. Math. 180:1 (1997), 125-133. MR 98k:32034 Zbl 0898.32015

[Nehari 1949] Z. Nehari, "The Schwarzian derivative and schlicht functions", Bull. Amer. Math. Soc. 55 (1949), 545-551. MR 10,696e Zbl 0035.05104

[Oda 1974] T. Oda, "On Schwarzian derivatives in several variables”, Sūrikaisekikenkyūsho Kōkyūroku (RIMS, Kyoto) 226 (1974). In Japanese.

[Pfaltzgraff 1974] J. A. Pfaltzgraff, "Subordination chains and univalence of holomorphic mappings

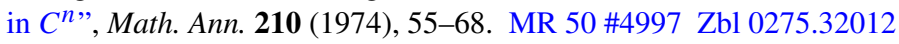

[Pfaltzgraff 1997] J. A. Pfaltzgraff, "Distortion of locally biholomorphic maps of the $n$-ball", Complex Variables Theory Appl. 33:1-4 (1997), 239-253. MR 99a:32030 Zbl 0912.32017

[Pfaltzgraff and Suffridge 2000] J. A. Pfaltzgraff and T. J. Suffridge, "Norm order and geometric properties of holomorphic mappings in $\mathbb{C}^{n}$ ", J. Anal. Math. 82 (2000), 285-313. MR 2001k:32028 Zbl 0978.32017

[Pommerenke 1964] C. Pommerenke, "Linear-invariante Familien analytischer Funktionen, I, II", Math. Ann. 155 (1964), 108-154 and 156 (1964), 226-262. MR 1513275 Zbl 0128.30105

[Yoshida 1976] M. Yoshida, "Canonical forms of some systems of linear partial differential equations”, Proc. Japan Acad. 52:9 (1976), 473-476. MR 54 \#13962 Zbl 0378.35013

[Yoshida 1984] M. Yoshida, "Orbifold-uniformizing differential equations", Math. Ann. 267 (1984), 125-142. MR 85j:14013 Zbl 0521.58052

Received March 7, 2005. Revised September 4, 2006. 
RODRIGO HERNÁNDEZ R.

UNIVERSIDAD ADOLFO IBÁÑEZ

FACUltad DE Ciencia y TeCNOlogía

AVENIDA LAS TORRES 2640

PEÑALOLÉN

CHILE

rodrigo.hernandez@uai.cl 